\title{
A 4bp-Insertion in the eya-Homologous Region (eyaHR) of EYA4 Causes Hearing Impairment in a Hungarian Family Linked to DFNA10
}

\author{
Markus Pfister, ${ }^{1}$ Tímea Tóth, ${ }^{2}$ Holger Thiele, ${ }^{3,5}$ Birgit Haack, ${ }^{1,4}$ Nikolaus Blin, ${ }^{4}$ Hans-Peter Zenner, ${ }^{1}$ \\ István Sziklai, ${ }^{3}$ Peter Nürnberg, ${ }^{3,5}$ Susan Kupka ${ }^{1,4}$ \\ ${ }^{1}$ Department of Otolaryngology, University of Tübingen, Tübingen, Germany \\ ${ }^{2}$ Medical and Health Science Center, Department of Otolaryngology, University of Debrecen, Hungary \\ ${ }^{3}$ Gene Mapping Center, Max Delbrück Center for Molecular Medicine, Berlin-Buch, Germany \\ ${ }^{4}$ Department of Anthropology and Human Genetics, University of Tübingen, Tübingen, Germany \\ ${ }^{5}$ Institute for Medical Genetics, Charité, Humboldt University, Berlin, Germany \\ Accepted August 23, 2002
}

\begin{abstract}
Background: Hereditary hearing impairment (HHI) is a heterogeneous class of disorders that shows various patterns of inheritance and involves a multitude of different genes. Mutations in the EYA4 gene are responsible for postlingual, progressive, autosomal dominant hearing loss at the DFNA10 locus. EYA4 is orthologous to the Drosophila gene eya ("eyes absent"), a key regulator of eye formation. EYA4 plays an important role in several developmental processes.

Material and Methods: Here we report a Hungarian family displaying sensorineural, progressive hearing impairment. The family comprising four generations with 11 affected and 8 unaffected members was subjected to genome-wide linkage analysis and candidate gene sequencing.
\end{abstract}

Results: By linkage analysis, the chromosomal region 6q22.3 was shown to segregate with the disease. Mutation analysis of the EYA4 gene, which maps to 6q22.3, revealed an insertion of $4 \mathrm{bp}$ (1558insTTTG) in all affected family members. This insertion creates a frameshift and results in a stop codon at position 379 . Hence, nearly the complete "eya homologous region" (eyaHR), which is essential for the protein function, would be deleted in the mutant EYA4 protein if the transcription were found to be stable.

Conclusions: This family is the third one linked to DFNA10 and revealing a mutation in the EYA4 gene. In all three families, the mutations are localized in different regions of the eyaHR, suggesting that this protein contains several functional subregions with different tissue-specific importance.

\section{Introduction}

Nonsyndromic severe to profound neurosensory hearing impairment (NSHL) is one of the most common human sensory disorder, affecting 1 in 1000 children with at least $60 \%$ of cases being inherited $(1,2)$. The mode of inheritance of nonsyndromic hearing disorders can be distinguished in autosomal dominant (10-15\%, DFNA), autosomal recessive $(70 \%, \mathrm{DFNB}), \mathrm{X}$-linked $(1-3 \%, \mathrm{DFN})$, and mitochondrial forms. NSHL accounts for up to $70 \%$ of all inherited sensorineural hearing defects. To date, 29 genes are known to play a role in NSHL (3).

Recently, Wayne et al. (4) showed the involvement of EYA4, a transcriptional activator and member of the vertebrate EYA family, in the development of DFNA10 in one Belgian and one American family. EYA4 is a ortholog to the Drosophila gene eya ("eyes absent"), which is involved in the formation of compound eyes (5). Flies with loss-of-function

Correspondence and reprint requests should be addressed to: Susan Kupka, University of Tübingen, Department of Otolaryngology, Molecular Genetics, Elfriede-Aulhorn-Str. 5, 72076 Tübingen, Germany. Phone: (49) 7071-29-88168; fax: (49) 7071-29-3311; e-mail: susankupka@yahoo.de. mutations of this gene develop no eyes. The human EYA4 encodes a transcriptional activator that interacts with members of the SIX and DACH protein families in a conserved network regulating early embryonic development. The protein contains a large, highly conserved C-terminal domain, the eya homology domain, and an alpha helical domain forming a leucine zipper (5).

Wayne et al. (4) found two EYA4 isoforms expressed in human fetal cochlear cDNA. In the same study, they identified mutations in the EYA4 gene that were responsible for postlingual, progressive, autosomal dominant hearing loss at the DFNA10 locus.

Here we present a third family with sensorineural hearing impairment also showing linkage to DFNA10 and a mutated EYA4 gene.

\section{Materials and Methods Patients}

The family is of Northeast Hungarian origin. The patients were recruited from the Department of Otolaryngology, University of Debrecen. To determine the etiology of hearing impairment, a detailed 
otolaryngologic examination was performed with the affected individuals and their unaffected relatives. The family comprised four generations and included 11 affected and 8 unaffected family members. According to the approval of the Ethics Comittee of the University of Debrecen, written informed consent was obtained from all participants and from parents of patients younger than 18 years.

\section{Audiologic Methods}

All family members underwent otoscopic and audiometric examinations by using age-appropriate methods. Threshold audiograms were obtained after otoscopic examination with pure-tone audiometry in a sound-treated room according to current clinical standards. We used air- and bone-conduction at 125 , 250, 500, 1000, 2000, 4000, and $8000 \mathrm{~Hz}$ for all affected participants. The audiometric configuration was classified based on the definitions of the European Work Group on Genetics of Hearing Impairment.

\section{Genotyping}

Genomic DNA was extracted from peripheral blood lymphocytes by standard techniques. Individuals were genotyped in a genomewide linkage analysis using 384 microsatellite markers with an average spacing of $11 \mathrm{cM}$. PCR reactions were performed using manufacturers' protocols. Semiautomated genotyping was performed by a MegaBACE- 1000 analysis system. Data were analyzed by Genetic Profiler Software 1.5. Two-point LOD score calculation was performed with the LINKAGE v5.2 program package (6). Most likely haplotypes were constructed with Simwalk2 v2.82 (7).

\section{Sequencing}

Sequencing was performed using primers described elsewhere (4). PCR products were gel extracted (Gel Extraction Kit, Qiagen) and sequenced with corresponding primers on an ABI 377 automated fluorescent sequencer machine. Detected mutations were confirmed at least two times and on both DNA strands. Sequences were compared with NCBIAccession number 13642856 using the DNAsis software (MWG).

\section{Results}

\section{Clinical Data}

The hearing disorder was sensorineural, progressive, and bilateral in all affected family members. At onset, hearing impairment was detected at the midand low-frequencies, degrading to a profound hearing impairment involving all frequencies.

\section{Linkage Analysis}

Microsatellite analysis revealed significant linkage of marker D6S1009 with the disease phenotype (max. two-point LOD score $Z_{\max }=4.73$ at $\Theta_{\max }=$ 0.00). By haplotype analysis, a critical interval of $36.8 \mathrm{cM}$ was determined between markers D6S262 and D6S305, which corresponds to the chromosomal region $6 \mathrm{q} 23.2-\mathrm{q} 26$ and contains the candidate gene EYA4 (Fig. 1).

\section{Sequencing}

Sequencing of all 21 EYA4 exons revealed an insertion of four bases (TTTG) in exon 13 (1558insTTTG) (Fig. 2). This insertion was detected in all affected family members (data not shown). Mutation 1558 insTTTG causes a frameshift beginning in codon 373 , followed by amino acid substitutions and a premature termination codon (PTC) at position 379. This PTC is likely to cause degradation of the mutant transcript by nonsense-mediated decay (5), otherwise it would result in a nearly complete deletion of the eya homologous region of EYA4.

\section{Discussion}

Autosomal dominant inherited hearing impairment is a genetically heterogenous disorder. So far, 41 chromosomal loci have been linked to this disease and 17 genes have been reported (3). Recently, Wayne et al. (4) identified mutations in the EYA4 gene that were responsible for postlingual, progressive, autosomal dominant loss at the DFNA10 locus. EYA4 is orthologous to the Drosophila gene eya ("eyes absent"), and is localized on 6q23 (6). The EYA4 gene consists of 21 exons, of which some are alternatively spliced creating several isoforms. The encoded protein contains a highly conserved 271 amino acid C-terminus called the eya-homologous region (eyaHR, eya domain) and a more divergent proline-serine-threonine (PST)-rich transactivation domain at the $\mathrm{N}$-terminus (6).

We report a DFNA10 family displaying sensorineural, progressive hearing impairment and linkage to $6 \mathrm{q} 23$. The results of the detailed audiometric analysis of a Belgian DFNA10 family coincide with our clinical findings (9). To our knowledge, this is the third DFNA10 family revealing a mutation in the EYA4 gene. The detected insertion of $4 \mathrm{bp}$ (1558insTTTG) creates a frameshift and results in a PTC at position 379 . The effect is either a complete degradation of the mutant messenger or a nearly complete deletion of the eyaHR in the EYA4 protein. The eyaHR is essential for members of the EYA protein family regarding their interaction with PAX, SIX, and DACH proteins in a genetic network which is conserved across species (10). First described in Drosophila as a key regulator of eye formation, this network and its function in several developmental processes had also been demonstrated to play an important role in vertebrates. Drosophila eya plays a critical role in morphogenesis of a number of tissues separately, during early eye formation (11). By analyzing Drosophila eya gene mutations 


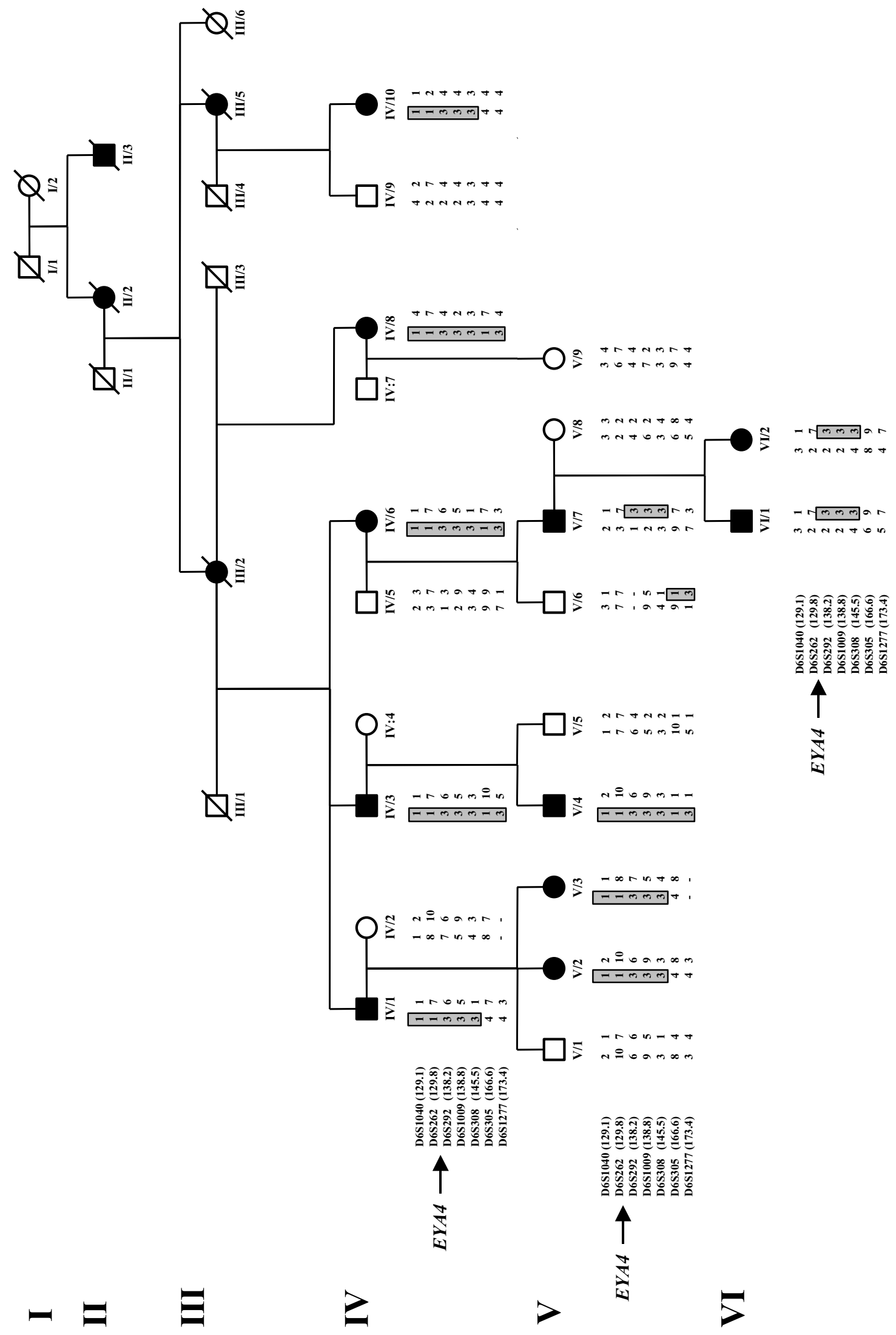


$\mathbf{A}$

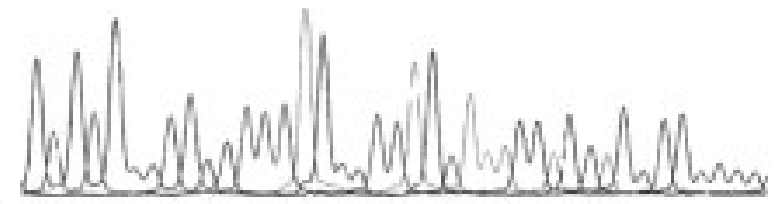

allek I GTTTGTCTGGGATTTGGATGAAACCATC.allele 2 GTTTGTCTGGGATTTGGATGAAACCATC.

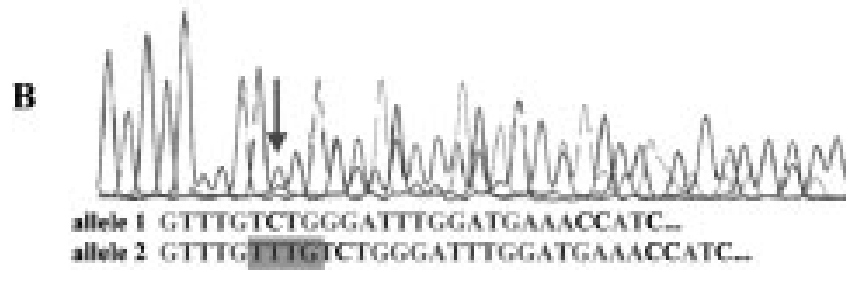

Fig. 2. Part of the sequence of EYA4 exon 13. (A) Nonaffected family member (V:1) displaying two normal alleles. (B) Affected family member (V:2) showing the heterozygote 4-bp insertion.

Bui et al. (12) showed that the loss of the entire eya domain results in eya inactivity, whereas alleles with truncations within the eya domain display partial function.

Recently, Heanue et al. (13) studied expression of the Dach/Pax/Eya network in mice and chicken. They showed that DachI and Eyal expression overlap in the developing ear and Pax2 and Eyal are required for normal ear development and they suggested that Drosophila Pax/Eya/Dach network may be evolutionarily conserved such that Pax genes, Eyal, and Dachl may function together in vertebrates to regulate neural development.

By studying eyal-deficient mice, Xu et al. (14) showed that eyal controls critical, early inductive signaling events involved in ear and kidney formation. Eyal heterozygotes $(+/-)$ showed renal abnormalities and a conductive hearing loss similar to human branchiootorenal dysplasia (BOR; OMIM 113650 ) syndrome, which is caused by mutations in EYA1. Eyal homozygotes $(-/-)$ lacked ears and kidneys due to defective inductive tissue interactions and apoptotic regression of the organ primordia. Inner ear development in Eyal null mice arrested at the otic vesicle stage, and all components of the inner ear and specific cranial sensory ganglia failed to form. The authors concluded that the evolutionary conserved pax-eya-six regulatory hierarchy is used in mammalian inner ear and kidney development (14).

So far, all three EYA4 mutations detected in DFNA10 families result in PTCs, presumably entailing nonsense-mediated decay of the mRNA (5) or, alternatively, deletions of parts of the eyaHR. Based on the observations mentioned, this region is of exceptional importance for the function of the protein. Therefore, it would not be surprising that, even if the mutant proteins were present in the cells, interrupting mutations within the eyaHR would lead to haploinsufficiency. Interestingly, the phenotype is obviously not depending on the position of the PTC which is commensurate with an instable mutant messenger. In our Hungarian family, and in the American family, almost the complete eyaHR is deleted, whereas in the Belgian family only a small part at the $\mathrm{C}$-terminus is absent (4). These observations support the presumption that the eyaHR contains several functional subregions with different tissue-specific importance. This would also serve as an explanation for the limited phenotype of DFNA10, showing no congenital abnormalities, despite the wide range of expression in early embryogenesis.

In addition to their developmental functions, members of the eya gene family were shown to act as a pro-apoptotic signal by Clark et al. (15). When overexpressed eya can directly activate the apoptotic program, and this function is conserved between fly, mouse, and human eya proteins. Eya-induced cell death has many features typical for apoptosis, including plasma and mitochondrial membrane changes and caspase activities. Furthermore, eya appears to induce apoptosis by triggering both caspase-dependent and caspase-independent pathways (15). Caspase-dependent apoptosis occurs in the inner ear, suggesting an important role in the functioning of the auditory system. Caspase-3 knockout mice show progressive severe hearing impairment, hyperplasia of supporting cells, and degeneration of sensory hair cells $(16,17)$. Progressive hearing impairment is a characteristic feature in DFNA10-linked families. Therefore, the apoptotic function of EYA4 may be relevant for the hearing process. Thus mutations within the EYA4 gene may not only provoke hearing impairment due to developmental failures but also because of apoptotic deficiencies.

\section{Acknowledgments}

This study was supported by grants of Else-KrönerFresenius-Stiftung, the Ministry of Education, Department for Research in Hungary (TeT 40/2000), and the Hungarian Basic Research Foundation (OTKAT037255) and Dr. Karl Kuhn-Stiftung. We thank all patients for their cooperation in the study.

\section{References}

1. Fraser GR. (1971) The genetics of congenital deafness. Otolaryngol. Clin. North Am. 4: 227-247.

2. Morton NE. (1991) Genetic epidemiology of hearing impairment. Ann N Y Acad Sci 630: 16-31.

3. Van Camp G, Smith RJ. Hereditary hearing loss homepage. Available from: URL: http://dnalab-www.uia.ac.be/dnalab/ hhh/. Retrieved August, 2002.

4. Wayne S, Robertson NG, DeClau F, et al. (2001) Mutations in the transcriptional activator EYA4 cause late-onset deafness at the DFNA10 locus. Hum. Mol. Genet. 10: 195-200.

5. Hentze MW, Kulozik AE. (1999) A perfect message: RNA surveillance and nonsense-mediated decay. Cell. 96: 307-310.

6. Borsani G, DeGrandi A, Ballabio A, et al. (1999) EYA4, a novel vertebrate gene related to Drosophila eyes absent. Hum. Mol. Genet. 8: 11-23. 
7. Lathrop G, Lalouel J. (1984) Easy calculations of lod scores and genetic risks on small computers. Am. J. Hum. Genet. 36: 460-465.

8. Sobel E, Lange K. (1996) Descent graphs in pedigree analysis: applications to haplotyping, locationscores, and markersharing statistics. Am. J. Hum. Genet. 58: 1323-1337.

9. Verstreken M, DeClau F, Schatteman I, et al. (2000) Audiometric analysis of a Belgian family linked to the DFNA10 locus. Am. J. Otolaryngol. 21: 675-681.

10. Heanue TA, Reshef R, Davis RJ, et al. (1999) Synergistic regulation of vertebrate muscle development by Dach2, Eya2, and Sixl, homologs of genes required for Drosophila eye formation. Genes Dev. 13: 3231-3243.

11. Bonini NM, Leiserson WM, Benzer S. (1998) Multiple roles of the eyes absent gene in Drosophila. Dev. Biol. 196: 42-57.

12. Bui QT, Zimmerman JE, Liu H, Bonini NM. (2000) Molecular analysis of Drosophila eyes absent mutants reveals features of the conserved Eya domain. Genetics 155: 709-720.
13. Heanue TA, Davis RJ, Rowitch, et al. (2002) Dachl, a vertebrate homologue of Drosophila dachshund, is expressed in the developing eye and ear of both chick and mouse and is regulated independently of Pax and Eya genes. Mech. Dev. 111: 75-87.

14. Xu PX, Adams J, Peters H, Brown MC, Heaney S, Maas R. (1999) Eyal-deficient mice lack ears and kidneys and show abnormal apoptosis of organ primordia. Nat. Genet. 23: 113-117.

15. Clark SW, Fee BE, Cleveland JL. (2002) Misexpression of the eyes absent family triggers the apoptotic program. J. Biol. Chem. 277: 3560-3567.

16. Morishita T, Makishima T, Kaneko C, et al. (2001) Deafness due to degeneration of cochlea neurons in caspase-3-deficient mice. Biochem. Biophys. Res. Commun. 284: 142-149.

17. Takahashi K, Kamiya K, Urase K, et al. (2001) Caspase3-deficiency induces hyperplasia of supporting cells and degeneration of sensory cells resulting in the hearing loss. Brain Res. 894: 359-367. 